This item was submitted to Loughborough's Research Repository by the author.

Items in Figshare are protected by copyright, with all rights reserved, unless otherwise indicated.

\title{
Myoelectric stimulation on peroneal muscles with electrodes of the muscle belly size attached to the upper shank gives the best effect in resisting simulated ankle sprain motion
}

\section{PLEASE CITE THE PUBLISHED VERSION}

http://dx.doi.org/10.1016/j.jbiomech.2013.01.019

\section{PUBLISHER}

(C) Elsevier

\section{VERSION}

AM (Accepted Manuscript)

\section{PUBLISHER STATEMENT}

This work is made available according to the conditions of the Creative Commons Attribution-NonCommercialNoDerivatives 4.0 International (CC BY-NC-ND 4.0) licence. Full details of this licence are available at: https://creativecommons.org/licenses/by-nc-nd/4.0/

\section{LICENCE}

CC BY-NC-ND 4.0

\section{REPOSITORY RECORD}

Fong, Daniel Tik-Pui, Dan Wang, Vikki Wing-Shan Chu, and Kai-Ming Chan. 2019. "Myoelectric Stimulation on Peroneal Muscles with Electrodes of the Muscle Belly Size Attached to the Upper Shank Gives the Best Effect in Resisting Simulated Ankle Sprain Motion". figshare. https://hdl.handle.net/2134/21214. 


\section{Elsevier Editorial System(tm) for Journal of Biomechanics}

Manuscript Draft

Manuscript Number: BM-D-12-00115R1

Title: Myoelectric stimulation on peroneal muscles resists simulated ankle sprain motion

Article Type: Short Communication (max 1500 words)

Keywords: ankle ligamentous sprain, injury prevention, biomechanics

Corresponding Author: Dr Daniel Tik-Pui Fong, PhD

Corresponding Author's Institution: The Chinese University of Hong Kong

First Author: Daniel Tik-Pui Fong, PhD

Order of Authors: Daniel Tik-Pui Fong, PhD; Vikki Chu, MPhil; Kai-Ming Chan, MBBS

Abstract: The inadequate reaction time of the peroneal muscles in response to an incorrect foot contact event has been proposed as one of the aetiological factors contributing to ankle joint inversion injury. Thus, the current study aimed to investigate the efficacy of a myoelectric stimulation applied to the peroneal muscles in the prevention of a simulated ankle inversion trauma. Ten healthy male subjects performed simulated inversion and supination tests on a pair of mechanical sprain simulators. An electrical signal was delivered to the peroneal muscles of the subjects through a pair of electrode pads. The start of the stimulus was synchronized with the drop of the sprain simulator's platform. In order to determine the maximum delay time which the stimulus could still resist the simulated ankle sprain motion, different delay time were test $(0,5,10$, and $15 \mathrm{~ms})$. Together with the control trial (no stimulus), there were 5 testing conditions for both simulated inversion and supination test.

The effect was quantified by the drop of maximum ankle tilting angle and angular velocity, as determined by a motion analysis system with a standard laboratory procedure. Results showed that the myoelectric stimulation was effective in all conditions except the one with myoelectric stimulus delayed for $15 \mathrm{~ms}$ in simulated supination test. It is concluded that myoelectric stimulation on peroneal muscles could resist an ankle spraining motion. 


\section{Cover Letter}

Dear Editor of Journal of Biomechanics,

$\underline{\text { Re: Submission of an article titled "Myoelectric stimulation on peroneal muscles resists }}$ $\underline{\text { simulated ankle sprain motion" }}$

The mentioned manuscript was submitted as "Original Article" on $31^{\text {st }}$ Jan, 2012 (Ref: Ms. No. BM-D-12-00115). As suggested by the referee, we revised the manuscript as a "Short Communication".

We declare that each author were fully involved in the study and preparation of the manuscript and that the material within has not been and will not be submitted for publication elsewhere.

Daniel Tik-Pui FONG

Vikki Wing-Shan CHU

Kai-Ming CHAN

10 Apr, 2012 
-----Original Message-----

From: ees.bm.0.17e2d6.e5329162@eesmail.elsevier.com

[mailto:ees.bm.0.17e2d6.e5329162@eesmail.elsevier.com] On Behalf Of Journal of Biomechanics

Sent: Friday, March 16, 2012 11:43 PM

To: dfong@ort.cuhk.edu.hk

Subject: BM-D-12-00115 - Editor Decision

Ref.: Ms. No. BM-D-12-00115

Myoelectric stimulation on peroneal muscles resists simulated ankle sprain motion Journal of Biomechanics

Dear Dr Fong,

Thank you for your submission to the Journal of Biomechanics. After considering the enclosed reviews from our referees, I regret to inform you that our referee panel recommends against publication of your manuscript in its current form, although a revised manuscript may be resubmitted and considered after further review. Although it is obvious your manuscript represents considerable work, and the referees and I believe it to be relevant to the Journal, one of the referees raised several major issues that would need to be addressed prior to publication. Their comments are attached for your information.

In addition, please rewrite your results section so that each paragraph is led with a clear statement of a key result. Refer to Tables and figures parenthetically (rather than "Table 1 showed ...").

The Results section was rewritten.

Please note that in consideration of the authors' and the reviewers' time, I normally allow only one major revision; if the reviewers request another major revision, I regret that we will not be able to publish your manuscript. Unfortunately, we have been forced to decrease our acceptance rate significantly due to an increase in manuscript submissions.

To submit a revision, go to http://ees.elsevier.com/bm/ and log in as an Author. You will see a menu item called Submission Needing Revision. You will find your submission record there. Please update accordingly and submit your revised manuscript."

If you choose to submit a revised manuscript, please provide a list of points of how you have responded to the reviewers' suggestions with the revised manuscript, at your earliest convenience. If you do not wish to proceed, please let us know in order to complete our records. The maximum time 
allowed for revision is 8 weeks, after which the file on this manuscript will be closed. If you feel you need longer than this please contact me.

\section{Please note:}

* Any figures and tables should be included, even if these are unaltered.

* It is the author's responsibility to ensure that data presented in figures and tables agree with that provided in the text. Please cross check figures, tables and text carefully.

* Please double-check formatting of your references

* Please use your word processor to automatically number the lines of your manuscript

* Please provide a word count, from the Introduction through the Acknowledgments.

Thank you again for submitting to the Journal of Biomechanics. I look forward to receiving your revised manuscript.

Yours sincerely,

Stephen Piazza, Ph.D.

Associate Editor

Farshid Guilak, Ph.D.

Editor-in-Chief

Journal of Biomechanics 
Reviewers' comments:

Reviewer \#1:

GENERAL COMMENT: The authors have produced an interesting paper which is of importance to the biomechancis community and particularly those with an interest in ankle joint sprain neuromechanics. I feel that the manuscript is not as concisely written and well presented as other works from this group, and the English language needs significant revision in places. However with some work, the paper in my opinion is worthy of publication as a short communication.

The manuscript was rewritten as a short communication as suggested.

\section{SECTION: ABSTRACT}

LINE: The slow reaction time of peroneal muscles is an aetiology to ankle sprain injury which causes the failure of adaptation to incorrect foot landing. This study proposed the use of myoelectric stimulation on peroneal muscles to initiate quick contraction to prevent ankle sprain injury, and evaluated its effect by its performance in resisting simulated ankle sprain motions in a laboratory setting.

COMMENT: My suggestion would be to re-phrase Inadequate reaction time of the peroneal muscles in response to an incorrect foot contact event has been proposed as one of the aetiological factors contributing to ankle joint inversion injury. Thus, the current study aimed to investigate the efficacy of a myoelectric stimulation applied to the peroneal muscles in the prevention of a simulated ankle inversion trauma.

Changed as suggested

LINE: The delay time was set at 0, 5, 10 and $15 \mathrm{~ms}$ to determine the maximum delay from the start of the electrical trigger which the device could still resist the simulated ankle sprain motion.

COMMENT: This line needs also to be re-phrased.

The sentences was rewritten to "The start of the stimulus is synchronized with the drop of the sprain simulator's platform. In order to determine the maximum delay time which the stimulus could still resist the simulated ankle sprain motion, different delay time were test $(1,5,10$, and $15 \mathrm{~ms})$. Together with the control trial (no stimulus), there were 5 testing conditions for both simulated inversion and supination test."

LINE: The two most commonly suggested aetiologies are the incorrect foot positioning at landing which generates sudden and excessive ankle inversion or supination torque, and the slow reaction 
time of the peroneal muscles at the lateral aspect of the ankle to accommodate by resistive eversion or pronation torque (Fong et al, 2009).

COMMENT: I would suggest breaking up this sentence.

[1] The first idea to introduce is the role of an incorrect foot contact event. This can occur during landing from a jump or also during gait.

My suggestion would be to introduce this as follows: "One factor commonly reported to contribute to the ankle sprain injury mechanism and particularly in the case of chronic ankle instability is an inappropriate positioning of the foot prior to and at initial contact with the ground during gait, landing from a jump and other sporting activities."

Appropriate references might be:

Wright IC, Neptune RR, van den Bogert AJ, Nigg BM. The influence of foot positioning on ankle sprains. J Biomech. 2000 May;33(5):513-9.

Konradsen L, Voigt M. Inversion injury biomechanics in functional ankle instability: a cadaver study of simulated gait. Scand J Med Sci Sports. 2002 Dec;12(6):329-36.

Mok KM, Fong DT, Krosshaug T, Engebretsen L, Hung AS, Yung PS, Chan KM. Kinematics analysis of ankle inversion ligamentous sprain injuries in sports: 2 cases during the 2008 Beijing Olympics. Am J Sports Med. $2011 \mathrm{Jul} ; 39(7): 1548-52$.

Mok KM, Fong DT, Krosshaug T, Hung AS, Yung PS, Chan KM. An ankle joint model-based image-matching motion analysis technique. Gait Posture. 2011 May;34(1):71-5.

Delahunt E, Monaghan K, Caulfield B. Changes in lower limb kinematics, kinetics, and muscle activity in subjects with functional instability of the ankle joint during a single leg drop jump. J Orthop Res. 2006 Oct;24(10):1991-2000.

Delahunt E, Monaghan K, Caulfield B. Altered neuromuscular control and ankle joint kinematics during walking in subjects with functional instability of the ankle joint. Am J Sports Med. 2006 Dec;34(12):1970-6.

Delahunt E, Monaghan K, Caulfield B. Ankle function during hopping in subjects with functional instability of the ankle joint. Scand J Med Sci Sports. 2007 Dec;17(6):641-8.

Changed as suggested and 3 references Mok et al, 2011, Delahunt et al, 2006 and 2007 were added.

[2] Next the authors should introduce the rationale behind a feedforward and feedback deficit in peroneal activation.

My suggestion would be to introduce this as follows: "It has also been suggested that a deficit in peroneal feedforward and feedback neuromuscular response may contribute to inappropriate positioning of the foot prior to and at initial contact. Furthermore, a increased latency in the peroneal muscles could further contribute to the injury mechanism, whereby these muscles cannot react in a time efficient manner to prevent an inversion trauma."

Appropriate references would be: 
Delahunt E, Monaghan K, Caulfield B. Changes in lower limb kinematics, kinetics, and muscle activity in subjects with functional instability of the ankle joint during a single leg drop jump. J Orthop Res. 2006 Oct;24(10):1991-2000.

Fong DT, Chan YY, Mok KM, Yung PSh, Chan KM. Understanding acute ankle ligamentous sprain injury in sports. Sports Med Arthrosc Rehabil Ther Technol. 2009 Jul 30;1:14.

Changed as suggested and the suggested references were added

LINE: Orthopaedic sport medicine specialists and sport scientists are working on ankle sprain prevention by different measures, such as ankle muscle strength and endurance training (So et al, 1994), proprioception and neuromuscular training (Xu et al, 2004). On top of these training, prophylactic apparels such as brace and taping are also widely used for ankle sprain prevention (Cordova et al, 2007), however, it may restrict the ankle range of motion so does the performance (Hume et al, 1998).

COMMENT: This section is a little weak and should be improved. I think that it is sufficient to say that recent studies suggest that neuromuscular training protocols incorporating strength, postural stability and proprioceptive exercises are effective in reducing functional insufficiencies associated with ankle sprain. However, the optimal training protocol has yet to be designed. In the absence of such a protocol the investigation of novel technologies is warranted [this then leads into the anti-sprain shoes development section].

The authors need to incorporate some more up-to-date references regarding neuromuscular training protocols.

Changed as suggested and a up-to0date referneces was added

Holmes, A., Delahunt, E., 2009. Treatment of common deficits associated with chronic ankle instability. Sports medicine 39, 207-224.

LINE: A new idea of prophylactic apparel, an intelligent anti-sprain shoe, was proposed in 2006. It provides protection actively when a sprain risk is sensed. During unharmed condition, it just likes a normal sport shoe, allows full range of ankle motion (Chan, 2006).

COMMENT: Please re-read to improve the English of these two sentences. Also the authros should use inversion sprain at all times, as we are interested in lateral ligament complex injury.

The sentences were rewritten to "In 2006, Chan proposed a new idea of prophylactic apparel - an intelligent anti-inversion-sprain shoe. It detects the foot motion continuously. Once hazardous motion is detected, a corrective mechanism will be activated to correct the landing motion (Chan 2006)."

LINE: Each subject performed five trials of simulated inversion test and five trials of simulated supination test.

COMMENT: Please improve the English here. 
This sentence was deleted

LINE: The voltage was gradually increased until the subject became unbearable. It was about $110 \mathrm{~V}$ $130 \mathrm{~V}$.

COMMENT: This should be "the voltage was gradually increased until it beacme intolerable to the subject (range 110 - 130V).

Changed as suggested

LINE: Control trial with no myoelectric stimulation being delivered during the simulated spraining test was collected for comparison.

COMMENT: This does not make sense. Please revise.

The sentencs was rewritten to "Three trials were preformed for each delay time in simulated inversion and supination test respectively. Average value was used for analysis. Subject also preformed 3 control trials which is simulated spraining test without myoelectric stimulation."

\section{STATISTICAL ANALYSIS:}

COMMENT: In this section it would be advisable for the authors to outline the sepcific independent varaibles and the dependent variables. What was the correlation among the dependent variables (max heel tilt, max heel tilt velocity)? If the correlation is weak or stong it may be a better option to run two seperate one-way repeated measures ANOVA (one for inversion and one for supination). In this case the dependent varaibles would be max heel tilt, max heel tilt velocity, while the independent variable would be condition (with 5 levels - represented by control, 0, 5, 10 and $15 \mathrm{~ms}$ ). the authors would be able to present the main effect reporting Wilks Lambda, F value, $\mathrm{p}$ value and eta squared value. Then post-hoc analysis would be conducted with the results presented in a table.

The statistic was redone as suggested. Since the maximu tilting angle and velocity is hight correlated (Pearson correlation > 0.7), two seperate one-way repeated measures ANOVA (one for inversion and one for supination) was used.

LINE: Both the maximum heel tilting angle and angular velocity did not significant differ from that in control condition.

COMMENT: I do not understand this line. Please clarify.

The result section was rewritten.

TABLE: Presumably there should be reference to (b) Supination? Reference to (b) Supination was added in all tables.

DISCUSSION:

COMMENT: All is clear and makes sense. 
Reviewer \#2:

I think this is an important work and should be published. 


\section{$\underline{\text { Title page }}$}

Title:

Myoelectric stimulation on peroneal muscles resists simulated ankle sprain motion

\section{Authors:}

Daniel Tik-Pui FONG ${ }^{1,2}, \mathrm{PhD}$

Vikki Wing-Shan $\mathrm{CHU}^{1}$, MPhil

Kai-Ming $\mathrm{CHAN}^{1,2}$, MBBS

\section{Affiliations:}

${ }^{1}$ Department of Orthopaedics and Traumatology, Prince of Wales Hospital, Faculty of Medicine, The Chinese University of Hong Kong, Hong Kong, CHINA

${ }^{2}$ The Hong Kong Jockey Club Sports Medicine and Health Sciences Centre, Faculty of Medicine, The Chinese University of Hong Kong, Hong Kong, CHINA

\section{Corresponding author:}

Dr Daniel Tik-Pui FONG

Email: dfong@ort.cuhk.edu.hk

Phone: (852) 26323535

Fax: (852) 26463020

Address: Department of Orthopaedics and Traumatology, Prince of Wales Hospital, Faculty of Medicine, The Chinese University of Hong Kong, Hong Kong, CHINA.

Keywords: ankle ligamentous sprain, injury prevention, biomechanics

Word count: 1497 


\begin{abstract}
$\underline{\text { Abstract }}$
The inadequate reaction time of the peroneal muscles in response to an incorrect foot contact event has been proposed as one of the aetiological factors contributing to ankle joint inversion injury. Thus, the current study aimed to investigate the efficacy of a myoelectric stimulation applied to the peroneal muscles in the prevention of a simulated ankle inversion trauma. Ten healthy male subjects performed simulated inversion and supination tests on a pair of mechanical sprain simulators. An electrical signal was delivered to the peroneal muscles of the subjects through a pair of electrode pads. The start of the stimulus was synchronized with the drop of the sprain simulator's platform. In order to determine the maximum delay time which the stimulus could still resist the simulated ankle sprain motion, different delay time were test $(0,5,10$, and $15 \mathrm{~ms})$. Together with the control trial (no stimulus), there were 5 testing conditions for both simulated inversion and supination test.
\end{abstract}

The effect was quantified by the drop of maximum ankle tilting angle and angular velocity, as determined by a motion analysis system with a standard laboratory procedure. Results showed that the myoelectric stimulation was effective in all conditions except the one with myoelectric stimulus delayed for $15 \mathrm{~ms}$ in simulated supination test. It is concluded that myoelectric stimulation on peroneal muscles could resist an ankle spraining motion. 
1

2

\section{Introduction}

Ankle sprain is one of the most common sport-related injuries (Fong et al, 2007; Fong et al, 2008). One factor commonly reported to contribute to the ankle sprain injury

mechanism and particularly in the case of chronic ankle instability is an inappropriate positioning of the foot prior to and at initial contact with the ground during gait, landing from a jump and other sporting activities (Mok et al, 2011, Delahunt et al, 2006 and 2007). It has also been suggested that a deficit in peroneal feedforward and feedback neuromuscular response may contribute to inappropriate positioning of the foot prior to and at initial contact. Furthermore, an increased latency in the peroneal muscles could further contribute to the injury mechanism, whereby these muscles cannot react in a time efficient manner to prevent an inversion trauma (Delahunt et al, 2006 and Fong et al, 2009).

Recent studies suggest that neuromuscular training protocols incorporating strength, postural stability and proprioceptive exercises are effective in reducing functional insufficiencies associated with ankle sprain (Holmes 2009). However, the optimal

training protocol has yet to be designed. In the absence of such a protocol the investigation of novel technologies is warranted. In 2006, Chan proposed a new idea of prophylactic apparel - an intelligent anti-inversion-sprain shoe. It detects the foot motion continuously. Once hazardous motion is detected, a corrective mechanism will be 
21 activated to correct the landing motion (Chan 2006).

23 One possible corrective mechanism for this intelligent shoe is to deliver a myoelectric

24 stimulation to the peroneal muscles to trigger quick reflex contraction. Such technique,

25 named "functional electrical stimulation", has been adopted in rehabilitation settings and

26 motor control research (Sabut et al, 2010). In our approach, the rationale is to utilize its

27 function and quick reaction to initiate peroneal muscle contraction and generate the

28 subsequent ankle joint pronation torque within 21-25ms (Ginz et al, 2004). The stimulus

29 could then take over the role of the slower peroneal muscles which react within $60-90 \mathrm{~ms}$

30 to resist the sudden ankle torque happening within $40-50 \mathrm{~ms}$ after the start of an ankle

31 joint inversion event (Fong et al, 2009).

33 The purpose of this study is to verify the action of artificial myoelectric stimulation of the

34 peroneal muscles and the appropriate timing necessary to reduce ankle inversion and

35 supination ranges during simulated sprain motion.

37 Materials and methods

38 Ten recreational male athletes (age $=22.6 \pm 2.4$ year, height $=1.72 \pm 0.04 \mathrm{~m}$, body

39 mass $=68.1 \pm 8.0 \mathrm{~kg}$ ) were recruited. The university ethics committee approved the study. A 
40 pair of mechanical sprain simulators was used to simulate inversion and supination

41 motion (Chan et al, 2008). In each trial, the subject stood with his body weight evenly

42 distributed on both platforms. One of the platform fell suddenly to a 30-degree tilted

43 orientation without prior notice to the subject.

45 A battery-powered myoelectric stimulation device was fabricated by the university

46 electronics services unit by modifying a previous design (Thorsen et al, 2009), with

47 adjustable voltage magnitude, activation time, and delay time of the stimulus from the

time of platform falls. The device was synchronized with the fall of the platform. A pair

of electrode pads (Panasonic EW4312P, Japan) was attached to the subject's peroneal

muscle belly. A myoelectric signal was delivered to the peroneal muscles to check if the

system was well equipped, as indicated by an involuntary ankle pronation motion right

after the delivery of myoelectric signal. The voltage was gradually increased until it

became intolerable to the subject (range $110 \mathrm{~V}-130 \mathrm{~V}$ ).

The delay time was set at $0,5,10$ and $15 \mathrm{~ms}$ in order to determine the maximum delay between the moments an ankle sprain starts to occur until the latest time which the device could still save the ankle joint. Since the electromechanical delay was reported to be 
could hardly catch up with a vigorous ankle sprain motion happening within 40-50ms.

The activation time was set to $500 \mathrm{~ms}$, which is enough to cover the duration of an ankle sprain motion. Three trials were preformed for each delay time in simulated inversion and supination test respectively. Average value was used for analysis. Subject also preformed 3 control trials which is simulated spraining test without myoelectric stimulation.

Twelve reflective markers ( $5 \mathrm{~mm}$ diameter) were attached to lateral fibula epicondyle, tibial tuberosity, lateral proximal shank, medial proximal shank, anterior distal shank, lateral distal shank, medial distal shank, posterior heel, lateral heel, medial heel, medial foot and dorsal foot. Marker coordinates were recorded by an optical motion analysis system (VICON, UK) at 500Hz. It were filtered by Generalized Cross-Validation package of Woltring with $15 \mathrm{~Hz}$ cut-off frequency (Woltring et al, 1986). A static calibration trial with the subject standing on the platforms in the anatomical position served as the offset position to determine the segment embedded axes of the shank and foot segment. The foot and shank segment were embedded with the Laboratory Coordinate System (LCS). A singular value decomposition method was employed to calculate the transformation from triad reference frame to anatomical shank and foot reference frame (Grood et al, 1983). Joint kinematics was deduced by the Joint Coordinate System (JCS) method (Soderkvist et al, 1993). Heel tilting angle was defined as the angle between the LCS vertical axis and 
78 foot transverse plane directional axis (Figure 2), and the heel tilting velocity was its

79 change with respect to time. The maximum measurements of these two parameters were

80 investigated. The data analysis was batch-processed by a customized Matlab program.

81

82 Statistical analysis

83 Shapiro-Wilk test was conducted to check the normality of each parameter in each

84 condition first. All parameter showed normality. Since maximum heel tilting angle and

85 angular velocity were highly correlated (Pearson correlation > 0.7), two separate one-way

86 repeated measures ANOVA were used for inversion and supination respectively. The

87 dependent variables choose for analysis were maximum heel tilting angle. The

88 independent variables were condition with 5 level (control, $0,5,10,15 \mathrm{~ms}$ delay time).

89

90 Post-hoc Bonferroni t-tests were then conducted to investigate which condition is differ.

91 Statistical significance was set at $\mathrm{p} \leq 0.05$.

\section{Results}

94 In both simulated inversion and supination tests, the maximum heel tilting angle dropped

95 from 18 degrees to 9-13 degrees, and the maximum heel tilting angular velocity dropped

96 from 200-250 degree/s to 140-170 degree/s. (Table 1) 
One way ANOVA with repeated measures results showed that there was different

between conditions in both inversion and supination test (Table 2). Post-hoc Bonferroni

t-tests further showed that the significant drop of the maximum heel tilting angle was

101 found between the conditions with and without stimulus, except $15 \mathrm{~ms}$ delay time. There

was no different among the trials with stimulus (Table 3).

\section{Discussion}

In this study, the myoelectric stimulation on peroneal muscles was found to be effective

in reducing the maximum heel tilting angle and angular velocity in the simulated ankle the fall. Compare with the case report of an accidental ankle sprain injury, the maximum

111 inversion velocity occurred at $30 \mathrm{~ms}$ after the foot strike (Fong et al, 2009). The time of

112 maximum velocity occurred later in simulated inversion motion than real injury case and

113 the value is much lower (253 deg/s in simulated motion and $632 \mathrm{deg} / \mathrm{s}$ in injury case).

114 This is because the motion in real injury case is much more vigorous, so larger angular

115 velocity can be reached within a short period of time. 
117 Our research team is developing an ankle sprain identification method utilizing motion

118 sensors to detect any hazardous ankle spraining motion (Chan et al, 2010; Chu et al,

119 2010). This result suggested that there is maximum $10 \mathrm{~ms}$ of time for the sensors to detect

120 a sprain motion, and to actuate the corrective system to protect the ankle joint in time.

121 The time limit may be even shorter in the real application since the injury motion is more

122 rigorous, hence more time or higher stimulation level is needed to resist/stop the motion.

123 In this study, the starting time was determined by the electrical trigger to initiate the fall

124 of the platform. In the future intelligent shoe, motion sensor is to be used as the trigger.

126 Conclusion

127 This study showed a good feasibility of delivering myoelectric stimulation on peroneal

128 muscles with $10 \mathrm{~ms}$ to resist sudden simulated ankle sprain motions. This corrective

129 mechanism could be implemented in the intelligent shoe to prevent ankle sprain injury.

131 Acknowledgement

132 This research project was made possible by the donation of The Hong Kong Jockey Club

133 Charities Trust. It is a project of The Hong Kong Research Institute of Textiles and

134 Apparel and is financially supported by the Innovation and Technology Fund from 
135 Innovation and Technology Commission, Hong Kong Special Administrative Region

136 Government, Project number: ITP/017/10TP. The authors acknowledge Mr Shee-Sun

137 Chiu of Electronics Services Unit of The Chinese University of Hong Kong for his help

138 to fabricate the myoelectric stimulation device.

139

140 References

141 Chan, K.M., 2006. Ankle injuries in sports - what's new on the horizon? Journal of Medical Biomechanics 21(Supp)6-7.

143 Chan, Y.Y., Fong, D.T.P., Chung, M.M.L., Li, W.J., Liao, W.H., Yung, P.S.H., Chan, K.M.,

144 2010. Identification of ankle sprain motion from common sporting motions by foot

145 kinematics data. Journal of Biomechanics 43, 1965-69.

146 Chan, Y.Y., Fong, D.T.P., Yung, P.S.H., Fung, K.Y., Chan, K.M., 2008. A mechanical

147 supination sprain simulator for studying ankle supination sprain kinematics.

$148 \quad$ Journal of Biomechanics 41, 2571-74.

149 Chu, V.W.S., Fong, D.T.P., Chan, Y.Y., Yung, P.S.H., Fung, K.Y., Chan, K.M., 2010.

150 Differentiation of ankle sprain motion and common sporting motions by ankle

151 inversion velocity. Journal of Biomechanics 43, 2035-38.

152 Delahunt, E., Monaghan, K., Caulfield, B., 2006. Changes in lower limb kinematics,

153 kinetics, and muscle activity in subjects with functional instability of the ankle 
joint during a single leg drop jump. Journal of Orthopaedic Research 24, 1991-2000.

Delahunt, E., Monaghan, K., Caulfield, B., 2006. Altered neuromuscular control and ankle joint kinematics during walking in subjects with functional instability of the ankle joint. The American Journal of Sports Medicine 34, 1970-1976.

Delahunt, E., Monaghan, K., Caulfield, B., 2007. Ankle function during hopping in subjects with functional instability of the ankle joint. Scandinavian Journal of Medicine \& Science in Sports 17, 641-8.

Fong, D.T.P., Chan, Y.Y., Mok, K.M., Yung, P.S.H., Chan, K.M., 2009. Understanding acute ankle ligamentous sprain injury in sports. Sports Medicine, Arthroscopy, Rehabilitation, Therapy and Technology 1:14.

Fong, D.T.P., Hong, Y., Chan, L.K., Yung, P.S.H., Chan, K.M., 2007. A systematic review on ankle injury and ankle sprain in sports. Sports Medicine 37, 73-94.

Fong, D.T.P., Hong, Y., Shima, Y., Krosshaug, T., Yung, P.S.H., Chan, K.M., 2009. Biomechanics of supination ankle sprain - a case report of an accidental injury event in the laboratory. The American Journal of Sports Medicine 37, 822-27. ankle injuries attending an accident and emergency department. Injury 39, 1222-27. 

techniques on isometric skeletal muscle strength. British Journal of Anaesthesia 92, $367-72$.

175

176

177

178

179

180

Grood, E.S., Suntay, W.J., 1983. A joint coordinate system for the clinical description of three-dimensional motions: application to the knee. Journal of Biomechanical Engineering 105, 136-144.

Holmes, A., Delahunt, E., 2009. Treatment of common deficits associated with chronic ankle instability. Sports Medicine 39, 207-224.

Mok, K.M., Fong, D.T.P., Krosshaug, T., Engebretsen, L., Hung, A.S.L., Yung, P.S.H., Chan, K.M., 2011. Kinematics analysis of ankle inversion ligamentous sprain injuries in sports: 2 cases during the 2008 Beijing Olympics. The American Journal of Sports Medicine 39, 1548-1552.

Sabut, S.K., Sikdar, C., Mondal, R., Kumar, R., Mahadevappa, M., 2010. Restoration of gait and motor recovery by functional electrical stimulation therapy in persons with stroke. Disability and Rehabilitation 32, 1594-1603.

Soderkvist, I., Wedin, P.A., 1993. Determining the movements of the skeleton using well-configured markers. Journal of Biomechanics 26, 1473-77.

Thorsen, R., Ferrarin, M., 2009. Battery powered neuromuscular stimulator circuit for use during simultaneous recording of myoelectric signals. Medical Engineering \& Physics 31, 1032-37. 
192 Woltring, H.J., 1986. A Fortran package for generalized, cross-validatory spline

193 smoothing and differentiation. Advances in Engineering Software 8, 104-113. 


\section{Table legends}

196 Table 1 -Mean and SD of maximum heel tilting angle and angular velocity.

197 Table 2 - Results of two one-way ANOVA with repeated measures. Dependent variable

198 was maximum heel tilting angle.

199 Table 3 - Results of post-hoc Bonferrion test.

200

201 Figure legends

202 Figure 1 - The simulated ankle sprain test.

203 Figure 2 -Heel tilting angle was defined as the ankle between the Laboratory Coordinate

204 System (LCS) vertical axis and foot transverse plane directional axis. 


\section{Figure}

Click here to download high resolution image

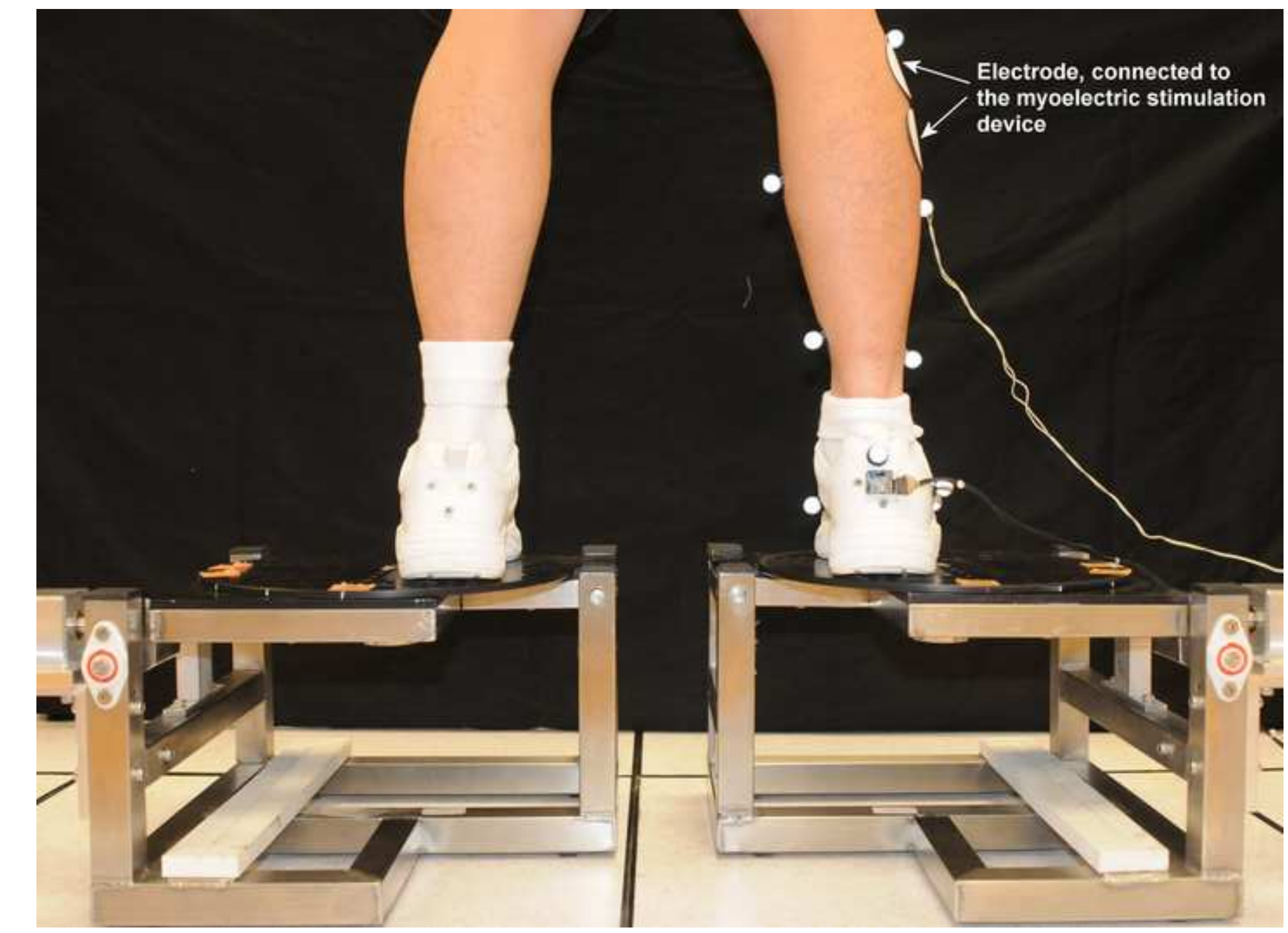




\section{Figure 2

Click here to download high resolution image

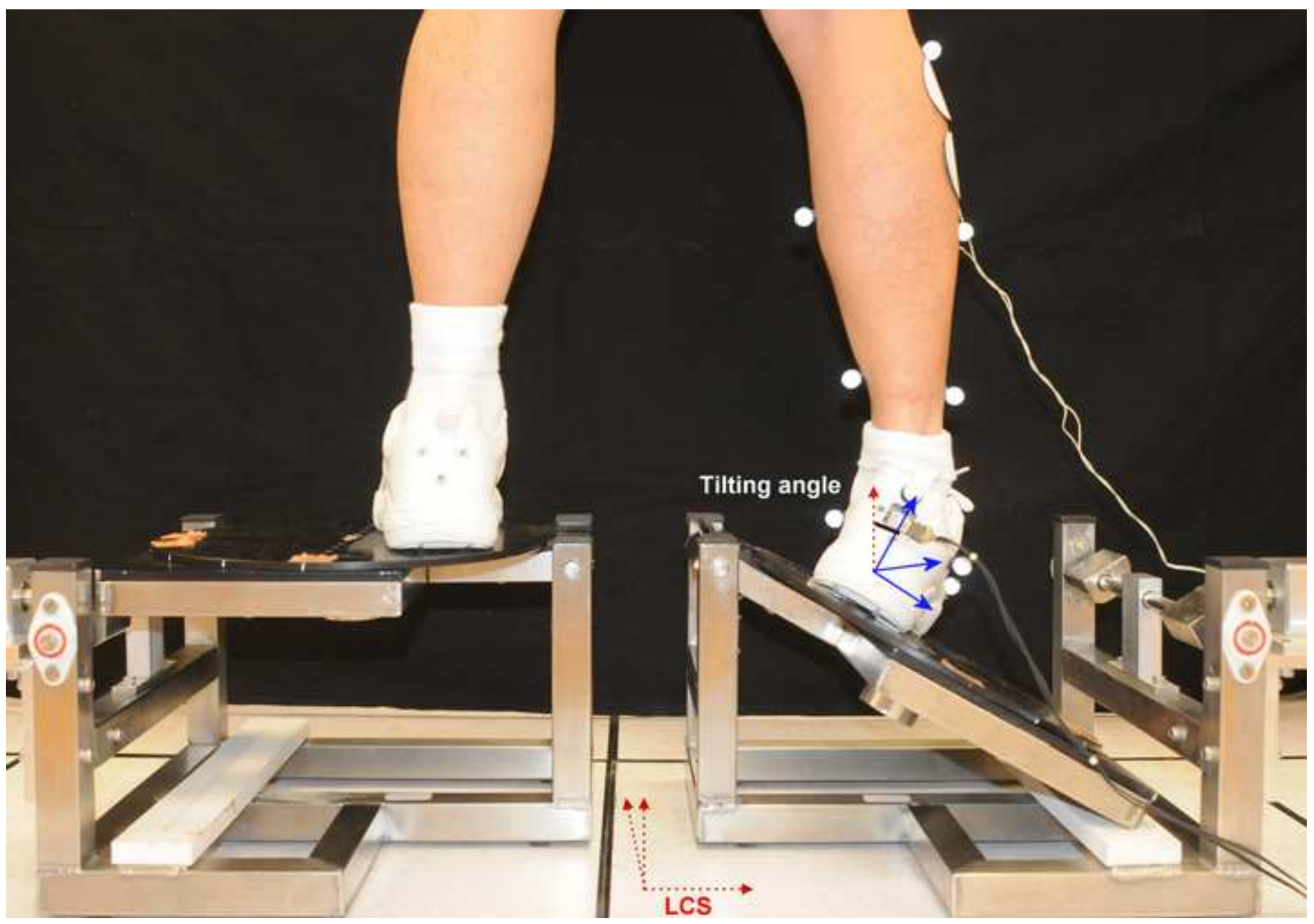


1 Table 1 - Mean and SD of maximum heel tilting angle and angular velocity.

\begin{tabular}{|c|c|c|c|c|c|}
\hline (a) Inversion test & $\begin{array}{c}\text { Maximum heel tilting angle } \\
\text { (degree) }\end{array}$ & $\begin{array}{c}\text { Maximum heel tilting } \\
\text { angular velocity (degree/s) }\end{array}$ & (b) Supination test & $\begin{array}{c}\text { Maximum heel tilting angle } \\
\text { (degree) }\end{array}$ & $\begin{array}{c}\text { Maximum heel tilting } \\
\text { angular velocity (degree/s) }\end{array}$ \\
\hline Control & $18.4(3.9)$ & $252.5(47.3)$ & Control & $17.7(6.3)$ & $206.3(69.2)$ \\
\hline Delay $=0 \mathrm{~ms}$ & $9.9(4.8)$ & $150.3(56.3)$ & Delay $=0 \mathrm{~ms}$ & $12.1(5.9)$ & $152.7(55.2)$ \\
\hline Delay $=5 \mathrm{~ms}$ & $9.4(5.7)$ & $144.2(70.4)$ & Delay $=5 \mathrm{~ms}$ & $13.6(6.4)$ & $158.1(63.6)$ \\
\hline Delay $=10 \mathrm{~ms}$ & $11.5(5.2)$ & $158.8(57.3)$ & Delay $=10 \mathrm{~ms}$ & $13.5(6.8)$ & $159.3(63.9)$ \\
\hline Delay $=15 \mathrm{~ms}$ & $12.5(6.0)$ & $172.6(66.2)$ & Delay $=15 \mathrm{~ms}$ & $12.7(6.8)$ & $157.2(58.3)$ \\
\hline
\end{tabular}

2

4 
Table 2 - Results of two one-way ANOVA with repeated measures. Dependent variable was maximum heel tilting angle.

\begin{tabular}{ccccc}
\hline & Wilks' Lambda & F-value & p-value & Partial Eta Squared \\
\hline (a) Inversion test & 0.111 & 12.042 & 0.005 & 0.8890. \\
(b) Supination test & 0.092 & 14.749 & 0.003 & 0.908 \\
\hline
\end{tabular}


Table 3 - Results of post-hoc Bonferrion test.

\begin{tabular}{|c|c|c|}
\hline & \multicolumn{2}{|c|}{ Maximum heel tilting angle (degree) } \\
\hline & $\begin{array}{l}\text { Bonferroni test } \\
\text { with control }^{\mathrm{b}}\end{array}$ & $\begin{array}{l}\text { Mean differences }(95 \% \\
\text { Confidence Interval })\end{array}$ \\
\hline \multicolumn{3}{|c|}{ (a) Inversion test } \\
\hline Control & - & 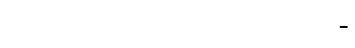 \\
\hline Delay $=0 \mathrm{~ms}$ & $0.001 *$ & $8.4(3.9-13.0)$ \\
\hline Delay $=5 \mathrm{~ms}$ & $0.003 *$ & $9.0(3.0-14.9)$ \\
\hline Delay $=10 \mathrm{~ms}$ & $0.002 *$ & $6.9(2.6-11.2)$ \\
\hline Delay $=15 \mathrm{~ms}$ & $0.040^{*}$ & $5.9(0.2-11.6)$ \\
\hline \multicolumn{3}{|c|}{ (b) Supination test } \\
\hline Control & - & - \\
\hline Delay $=0 \mathrm{~ms}$ & $0.002 *$ & $5.5(2.3-8.8)$ \\
\hline Delay $=5 \mathrm{~ms}$ & $0.001 *$ & $4.6(3.0-7.3)$ \\
\hline Delay $=10 \mathrm{~ms}$ & $0.046^{*}$ & $4.2(0.1-8.4)$ \\
\hline Delay $=15 \mathrm{~ms}$ & 0.240 & $4.9(-1.8-11.7)$ \\
\hline
\end{tabular}

In Bonferroni test, significant difference with $\mathrm{p}$ value less than 0.05 was denoted by an asterisk (*). 


\section{Conflict of interest}

Dear Editor of Journal of Biomechanics,

REF: Submission of manuscript titled "Myoelectric stimulation on peroneal muscles resists simulated ankle sprain motion".

The authors declare no financial and personal relationships with other people or organizations that could inappropriately influence this submitted work.

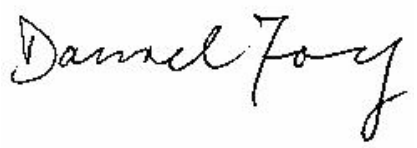

Daniel Tik-Pui FONG

Jan 30, 2012. 